\title{
Search for star cluster age gradients across spiral arms of three LEGUS disc galaxies
}

F. Shabani, ${ }^{1 \star}$ E. K. Grebel, ${ }^{1}$ A. Pasquali, ${ }^{1}$ E. D’Onghia, ${ }^{2,3}$ J. S. Gallagher, III, ${ }^{2}$ A. Adamo, ${ }^{4}$ M. Messa ${ }^{4}$ B. G. Elmegreen,${ }^{5}$ C. Dobbs,${ }^{6}$ D. A. Gouliermis,, 8 D. Calzetti, ${ }^{9}$ K. Grasha, ${ }^{9}$ D. M. Elmegreen, ${ }^{10}$ M. Cignoni, ${ }^{11,12,13}$ D. A. Dale,${ }^{14}$ A. Aloisi, ${ }^{15}$ L. J. Smith, ${ }^{16}$ M. Tosi, ${ }^{13}$ D. A. Thilker, ${ }^{17}$ J. C. Lee, ${ }^{15,18}$ E. Sabbi, ${ }^{15}$ H. Kim ${ }^{19}$ and A. Pellerin ${ }^{20}$

${ }^{1}$ Astronomisches Rechen-Institut, Zentrum für Astronomie der Universität Heidelberg, Mönchhofstr. 12-14, D-69120 Heidelberg, Germany

${ }^{2}$ Department of Astronomy, University of Wisconsin- Madison, 475 N. Charter Street, Madison, WI 53076-1582, USA

${ }^{3}$ Center for Computational Astrophysics, Flatiron Institute, 162 Fifth Avenue, New York, NY 10010, USA

${ }^{4}$ Department of Astronomy, The Oskar Klein Centre, Stockholm University, Stockholm, Sweden

${ }^{5}$ IBM Research Division, T.J. Watson Research Center, Yorktown Hts., NY, USA

${ }^{6}$ School of Physics and Astronomy, University of Exeter, Exeter, UK

${ }^{7}$ Institut für Theoretische Astrophysik, Zentrum für Astronomie der Universität Heidelberg, Albert-Ueberle-Str. 2, D-69120 Heidelberg, Germany

${ }^{8}$ Max Planck Institute for Astronomy, Königstuhl 17, D-69117 Heidelberg, Germany

${ }^{9}$ Department of Astronomy, University of Massachusetts - Amherst, Amherst, MA 01003, USA

${ }^{10}$ Department of Physics and Astronomy, Vassar College, Poughkeepsie, NY, USA

${ }^{11}$ Department of Physics, University of Pisa, Largo B. Pontecorvo 3, I-56127 Pisa, Italy

${ }^{12}$ INFN, Largo B. Pontecorvo 3, I-56127 Pisa, Italy

${ }^{13}$ INAF - Osservatorio Astrofisico e di Scienza dello Spazio, Bologna, Italy

${ }^{14}$ Department of Physics and Astronomy, University of Wyoming, Laramie, WY, USA

${ }^{15}$ Space Telescope Science Institute, Baltimore, MD, USA

${ }^{16}$ European Space Agency/Space Telescope Science Institute, Baltimore, MD, USA

${ }^{17}$ Department of Physics and Astronomy, The Johns Hopkins University, Baltimore, MD, USA

${ }^{18}$ Visiting Astronomer, Spitzer Science Center, Caltech. Pasadena, CA, USA

${ }^{19}$ Gemini Observatory, Casilla 603, La Serena, Chile

${ }^{20}$ Department of Physics and Astronomy, State University of New York at Geneseo, Geneseo, NY, USA

Accepted 2018 May 11. Received 2018 April 23; in original form 2018 January 13

\begin{abstract}
One of the main theories for explaining the formation of spiral arms in galaxies is the stationary density wave theory. This theory predicts the existence of an age gradient across the arms. We use the stellar cluster catalogues of the galaxies NGC 1566, M51a, and NGC 628 from the Legacy ExtraGalactic UV Survey (LEGUS) program. In order to test for the possible existence of an age sequence across the spiral arms, we quantified the azimuthal offset between star clusters of different ages in our target galaxies. We found that NGC 1566, a grand-design spiral galaxy with bisymmetric arms and a strong bar, shows a significant age gradient across the spiral arms that appear to be consistent with the prediction of the stationary density wave theory. In contrast, M51a with its two well-defined spiral arms and a weaker bar does not show an age gradient across the arms. In addition, a comparison with non-LEGUS star cluster catalogues for M51a yields similar results. We believe that the spiral structure of M51a is not the result of a stationary density wave with a fixed pattern speed. Instead, tidal interactions could be the dominant mechanism for the formation of spiral arms. We also found no offset in the azimuthal distribution of star clusters with different ages across the weak spiral arms of NGC 628.
\end{abstract}

Key words: galaxies: individual: NGC 1566, M51, NGC 628-galaxies: spiral-galaxies: structure. 


\section{INTRODUCTION}

Understanding how spiral patterns form in disc galaxies is a longstanding issue in astrophysics. Two of the most influential theories to explain the formation of spiral structure in disc galaxies are named stationary density wave theory and swing amplification. The stationary density wave theory poses that spiral arms are static density waves (Lindblad 1963; Lin \& Shu 1964). In this scenario, spiral arms are stationary and long-lived. The swing amplification proposes instead that spiral structure is the local amplification in a differentially rotating disc (Goldreich \& Lynden-Bell 1965; Julian \& Toomre 1966; Sellwood \& Carlberg 1984; Sellwood 2011; Elmegreen 2011; D’Onghia, Vogelsberger \& Hernquist 2013). According to this theory, indiviual spiral arms would fade away in one galactic year and should be considered transient features. Numerical experiments suggest that non-linear gravitational effects would make spiral arms fluctuate in density locally, but be statistically long-lived and self-perpetuating (D'Onghia et al. 2013).

To complicate the picture, there is the finding that many galaxies in the nearby universe are grand-design, bisymmetric spirals. These galaxies may show evidence of a galaxy companion, suggesting that the perturbations induced by tidal interactions could induce spiral features in discs by creating localized disturbances that grow by swing amplification (Kormendy \& Norman 1979; Bottema 2003; Gauthier, Dubinski \& Widrow 2006; D’Onghia et al. 2016; Pettitt, Tasker \& Wadsley 2016). Some studies have been devoted to explore galaxy models with bar-induced spiral structure (Contopoulos \& Papayannopoulos 1980) and spiral features explained by a manifold (Contopoulos \& Papayannopoulos 1980; Athanassoula 1992). It is also possible that a combination of these models is needed to describe the observed spiral structure. We refer the interested reader to comprehensive reviews of different theories of spiral structure in Dobbs \& Baba (2014) and to Shu (2016) for detailed explanations of the origin of spiral structure in stationary density wave theory.

The longevity of spiral structure can be tested observationally. In fact, in the stationary density wave theory, spiral arms are density waves moving with a single constant angular pattern speed. The angular speed of stars and gas equals the pattern speed at the corotation radius. Inside the corotation radius, material rotates faster than the spiral pattern. When the gas enters the higher density region of spiral arms, it may experience a shock which may lead to star formation (Roberts 1969). Consequently, the stars born in the molecular clouds in spiral arms eventually overtake the arms and move away from the spiral patterns as they age. This drift causes an age gradient across the spiral arms. If spiral arms have a constant angular speed, then we expect to find the youngest star clusters near the arm on the trailing side, and the oldest star clusters further away from the spiral arms inside the corotation radius (e.g. Martínez-García, González-Lópezlira \& Bruzual-A 2009). Outside the corotation radius, the spiral pattern moves faster than the gas and leads to the opposite age sequence.

Dobbs \& Pringle (2010) carried out numerical simulations of the age distribution of star clusters in four different spiral galaxy models, including a galaxy with a fixed pattern speed, a barred galaxy, a flocculent galaxy, and an interacting galaxy. The results of their simulations show that in a spiral galaxy with a constant pattern speed or in a barred galaxy, a clear age sequence across spiral arms from younger to older stars is expected. In the case of a flocculent spiral galaxy, no age gradient can be observed in their simulation. Also in the case of an interacting galaxy, a lack of an age gradient as a function of azimuthal distance from the spiral arms is predicted. A simulation of an isolated multiple-arm-barred spiral galaxy was performed by Grand, Kawata \& Cropper (2012), who explored the location of star particles as a function of age around the spiral arms. Their simulation takes into account radiative cooling and star formation. They found no significant spatial offset between star particles of different ages, suggesting that spiral arms in such a spiral galaxy are not consistent with the long-lived spiral arms predicted by the static or stationary density wave theory. In a recent numerical study, Dobbs et al. (2017) looked in detail at the spatial distribution of stars with different ages in an isolated grand-design spiral galaxy. They found that star clusters of different ages are all concentrated along the spiral arms without a clear age pattern.

A simple test of the stationary density wave theory consists of looking for a colour gradient from blue to red across spiral arms due to the progression of star formation. It is important to note that this method can be affected by the presence of dust. Several observational studies have tried to test the stationary density wave theory by looking for colour gradients across the spiral arms. In an early study of the $(B-V)$ colours and total star formation rates in a sample of spiral galaxies with and without grand design patterns, Elmegreen \& Elmegreen (1986) found no evidence for an excess of star formation due to the presence of a spiral density wave, and explained the blue spiral arm colours as a result of a greater compression of the gas compared to the old stars, with star formation following the gas. Martínez-García et al. (2009) studied the colour gradients across the spiral arms of $13 \mathrm{SA}$ and SAB galaxies. Ten galaxies in their sample present the expected colour gradient across their spiral arms.

A number of observational studies have used the age of stellar clusters in nearby galaxies as a tool to test the stationary density wave theory. Scheepmaker et al. (2009) studied the spatial distribution of 1580 stellar clusters in the interacting, grand-design spiral M51 a from Hubble Space Telescope (HST) UBVI photometry. They found no spatial offset between the azimuthal distribution of cluster samples of different age. Their results indicate that most of the young (age $<10 \mathrm{Myr}$ ) and old stellar clusters (age > $30 \mathrm{Myr}$ ) are located at the centres of the spiral arms. Kaleida \& Scowen (2010) also mapped the age of star clusters as a function of their location in M51a using HST data and found no clear pattern in the location of star clusters with respect to their age. Both above studies suggest that spiral arms are not stationary, at least for galaxies in tidal interaction with a companion. In order to study the spatial distribution of star-forming regions, Sánchez-Gil et al. (2011) produced an age map of six nearby grand-design and flocculent spiral galaxies. Only two grand-design spiral galaxies in their sample presented a stellar age sequence across the spiral arms as expected from stationary density wave theory.

In galaxies where spiral arms are long-lived and stationary as predicted by the static density wave theory, one would expect to find an angular offset among star formation and gas tracers of different age within spiral arms (Roberts 1969). The majority of observational studies of the spiral density wave scenario have tried to examine such an angular offset (Vogel, Kulkarni \& Scoville 1988; Rand 1995). Tamburro et al. (2008) detected an angular offset between $\mathrm{HI}$ (a tracer of the cold dense gas) and $24 \mu \mathrm{m}$ emission (a tracer of obscured star formation) in a sample of 14 nearby disc galaxies. An angular offset between $\mathrm{CO}$ (a tracer of molecular gas) and $\mathrm{H} \alpha$ (a tracer of young stars) was detected for 5 out of 13 spiral galaxies observed by Egusa et al. (2009). In another observational work, Foyle et al. (2011) tested the angular offset between different star formation and gas tracers including $\mathrm{HI}, \mathrm{H}_{2}, 24 \mu \mathrm{m}$, ultraviolet (UV, a tracer for unobscured young stars), and $3.6 \mu \mathrm{m}$ emission (a tracer of the underlying old stellar population) for 12 nearby disc galaxies. 
They detected no systematic trend between the different tracers. Similarly, Ferreras et al. (2012) found no significant angular offset between $\mathrm{H} \alpha$ and UV emission in NGC 4321. Louie, Koda \& Egusa (2013) found a large angular offset between $\mathrm{CO}$ and $\mathrm{H} \alpha$ in M51a while no significant offsets have been found between $\mathrm{HI}, 21 \mathrm{~cm}$, and $24 \mu \mathrm{m}$ emissions. These searches for offsets are based on the assumption that the different tracers represent a time sequence of the way a moving density wave interacts with gas and triggers star formation. Elmegreen et al. (2014) used the Spitzer Survey of Stellar Structure in Galaxies survey (Sheth et al. 2010) and discovered embedded clusters inside the dust lanes of several galaxies with spiral waves, suggesting that star formation can sometimes start quickly.

In a recent observational study, Schinnerer et al. (2017) carried out a detailed investigation of a spiral arm segment in M51a. They measured the radial offset of the star clusters of different ages ( $<3$ and 3-10 Myr) and star formation tracers (HII regions and $24 \mu \mathrm{m}$ ) from their nearest spiral arm. No obvious spatial offset between star clusters younger and older than $3 \mathrm{Myr}$ was found in M51a. They also found no clear trend in the radial offset of HII regions and $24 \mu \mathrm{m}$. Similarly, Chandar et al. (2017) compared the location of star clusters with different ages $(<6,6-30,30$ $100,100-400$, and $>400 \mathrm{Myr}$ ) with the spiral patterns traced by molecular gas, dust, young and old stars in M51a. They found cold molecular gas and dark dust lanes to be located along the inner edge of the arms while the outer edge is defined by the old stars (traced with $3.6 \mu \mathrm{m}$ ) and young star clusters. The observed sequence in the spiral arm of M51a is in agreement with the prediction from stationary density wave theory. Chandar et al. (2017) also measured the spatial offset between molecular gas, young $(<10 \mathrm{Myr})$ and old star clusters (100-400 Myr) in the inner (2.0-2.5 kpc) and outer (5.0-5.5 kpc) spiral arms in M51a. They found an azimuthal offset between the gas and star clusters in the inner spiral arm zone, which is consistent with the spiral density wave theory. In the outer spiral arms, the lack of such a spatial offset suggests that the outer spiral arms do not have a constant pattern speed and are not static. Chandar et al. (2017) found no star cluster age gradient along four gas spurs (perpendicular to the spiral arms) in M51a.

In conclusion, there have been numerous observational studies aiming to test the longevity of the spiral structure. In many cases, the conclusions show conflicting results and the nature of spiral arms is still an open question.

The main goal of this study is to test whether spiral arms in disc galaxies are static and long-lived or locally changing in density and locally transient. This work is based on the Legacy ExtraGalactic UV Survey (LEGUS) ${ }^{1}$ observations obtained with HST (Calzetti et al. 2015). The paper is organized as follows: the survey and the sample galaxies are described in Section 2. The selection of the star cluster samples is presented in Section 3. We investigate the spatial distribution together with clustering of the selected clusters in Section 4. In Section 5, we describe the results and analysis and how we measure the spatial offset of our star clusters across spiral arms. In Section 6, we discuss whether the two spiral arms of our target galaxies have the same nature. In Section 7, we use a non-LEGUS star cluster catalogue to measure the spatial offset of star clusters in M51a and we present our conclusions in Section 8 .

${ }^{1}$ https://legus.stsci.edu

\section{THE SAMPLE GALAXIES}

LEGUS is an HST Cycle 21 Treasury programme that has observed 50 nearby star-forming dwarf and spiral galaxies within $12 \mathrm{Mpc}$. High-resolution images of these galaxies were obtained with the UVIS channel of the Wide Field Camera Three (WFC3), supplemented with archival Advanced Camera for Surveys (ACS) imaging when available, in five broad-band filters, $N U V(F 275 W)$, $U(F 336 W), B(F 438 W), V(F 555 W)$, and $I(F 814 W)$. The pixel scale of these observations is 0.04 arcsec pixel ${ }^{-1}$. A description of the survey, the observations, the image processing, and the data reduction can be found in Calzetti et al. (2015).

Face-on spiral galaxies with prominent spiral structures are interesting candidates to study stationary density wave theory. Therefore, three face-on spiral galaxies, namely NGC 1566, M51a, and NGC 628 were selected from the LEGUS survey for our study. The morphology, distance, corotation radius, and the pattern speed of each galaxy are listed in Table 1. The UVIS and ACS footprints of the pointings (red and yellow boxes, respectively) overlaid on Digitized Sky Survey (DSS) images of the galaxies are shown in Fig. 1 together with their HST red, green, and blue colour composite mosaics.

\subsection{NGC 1566}

NGC 1566, the brightest member of the Dorado group, is a nearly face-on (inclination $=37.3^{\circ}$ ) barred grand-design spiral galaxy with strong spiral structures (Elmegreen et al. 2011). The distance of NGC 1566 in the literature is uncertain and varies between 5.5 and 21.3 Mpc. In this study, we revised the distance of $13.2 \mathrm{Mpc}$ listed in Calzetti et al. (2015) and adopted a distance of $18 \mathrm{Mpc}$ (Sabbi et al. 2018). NGC 1566 has been morphologically classified as an SABbc galaxy because of its intermediate-strength bar. It hosts a low-luminosity active galactic nucleus (Combes et al. 2014). The star formation rate and stellar mass of NGC 1566 are $2.0 \mathrm{M}_{\odot} \mathrm{yr}^{-1}$ and $2.7 \times 10^{10} \mathrm{M}_{\odot}$, respectively within the LEGUS field of view (Sabbi et al. 2018). Two sets of spiral arms can be observed in NGC 1566. The inner arms connect with the star-forming ring at $1.7 \mathrm{kpc}$ (Smajić et al. 2015), which is covered by the LEGUS field of view (see Fig. 1, top panel). The outer arms beyond 100 arcsec (corresponding to $8 \mathrm{kpc}$ ) are weaker and smoother than the inner arms.

\subsection{M51a}

M51a (NGC 5194) is a nearby, almost face-on (inclination $=22^{\circ}$ ) spiral galaxy located at a distance of 7.6 Mpc (Tonry et al. 2001). It is a grand-design spiral galaxy morphologically classified as SAc with strong spiral patterns (Elmegreen et al. 2011). M51a is interacting with a companion galaxy, M51b (NGC 5195). M51a has a star formation rate and a stellar mass of $6.9 \mathrm{M}_{\odot} \mathrm{yr}^{-1}$ and $2.4 \times 10^{10} \mathrm{M}_{\odot}$, respectively (Lee et al. 2009; Bothwell, Kennicutt \& Lee 2009). Five UVIS pointings in total were taken through LEGUS observations: four pointings cover the centre, the north-east, and the south-west regions of M51a, and one covers the companion galaxy M51b.

\subsection{NGC 628}

NGC 628 (M74) is the largest galaxy in its group. This nearby galaxy is seen almost face-on $\left(i=25.2^{\circ}\right)$ and is located at a distance of 9.9 Mpc (Olivares et al. 2010). It has no bulge (Cornett et al. 1994) and is classified as aN SAc spiral galaxy. Its star formation 
Table 1. Fundamental properties of our target galaxies.

\begin{tabular}{lcccccc}
\hline galaxy & Morphology & $D(\mathrm{Mpc})$ & $\mathrm{M}_{\star}\left(\mathrm{M}_{\odot}\right)$ & $\begin{array}{c}\text { SFR (UV) } \\
\left(\mathrm{M}_{\odot} \mathrm{yr}\right)\end{array}$ & $R_{\mathrm{cr}}(\mathrm{kpc})$ & $\begin{array}{c}\Omega_{\mathrm{p}} \\
\left(\mathrm{km} \mathrm{s}^{-1} \mathrm{kpc}^{-1}\right)\end{array}$ \\
\hline NGC 1566 & SABbc & 18 & $2.7 \times 10^{10}$ & 2.026 & 10.6 & $23 \pm 2$ \\
M51a & SAc & 7.6 & $2.4 \times 10^{10}$ & 6.88 & 5.5 & $38 \pm 7$ \\
NGC 628 & SAc & 9.9 & $1.1 \times 10^{10}$ & 3.6 & 7 & $32 \pm 2$ \\
\hline
\end{tabular}

Columns 1 and 2: galaxy name and morphological type as listed in the NASA Extragalactic Database (NED).

Column 3: distance.

Column 4: stellar mass obtained from the extinction-corrected $B$-band luminosity.

Column 5: star formation rate calculated from the GALEX far-UV, corrected for dust attenuation.

Column 6: corotation radius.

Column 7: pattern speed.

Column 8: references for the corotation radii and pattern speeds: 1 - Agüero, Díaz \& Bajaja (2004), 2 - Zimmer, Rand \& McGraw (2004), and 3 - Sakhibov \& Smirnov (2004).

rate and stellar mass obtained from the extinction-corrected $B$-band luminosity are $3.6 \mathrm{M}_{\odot} \mathrm{yr}^{-1}$ and $1.1 \times 10^{10} \mathrm{M}_{\odot}$, respectively (Lee et al. 2009; Bothwell et al. 2009). NGC 628 is a multiple-arm spiral galaxy (Elmegreen \& Elmegreen 1987) with two well-defined spiral arms. It has weaker spiral patterns than NGC 1566 and M51a (Elmegreen et al. 2011). The LEGUS UVIS observations of NGC 628 consist of one central and one east pointing that were combined into a single mosaic for the analysis.

\section{STELLAR CLUSTER SAMPLES}

\subsection{Selection from star cluster catalogues}

In this section, we provide a detailed explanation of the process adopted to select star cluster candidates in our target galaxies. A general description of the standard data reduction of the LEGUS sample can be found in Calzetti et al. (2015). A careful and detailed description of the cluster extraction, identification, classification, and photometry is given in Adamo et al. (2017) and Messa et al. (2018). Stellar cluster candidates were extracted with SEXTRACTOR (Bertin \& Arnouts 1996) in the five standard LEGUS filters. The resulting cluster candidate catalogues include sources with a $V$-band concentration index $(\mathrm{CI})^{2}$ larger than the CI of star-like sources, which are detected in at least two filters with a photometric error $\leq$ $0.3 \mathrm{mag}$. The photometry of sources in each filter was corrected for the Galactic foreground extinction (Schlafly \& Finkbeiner 2011). In order to derive the cluster physical properties such as age, mass, and extinction, the spectral energy distribution of the clusters was fitted with Yggdrasil stellar population models (Zackrisson et al. 2011). The uncertainties derived in the physical parameters of the star clusters are on average 0.1 dex (Adamo et al. 2017). For some of the LEGUS galaxies, star cluster properties were also estimated based on a Bayesian approach, using the Stochastically Lighting Up Galaxies (sLuG) code (da Silva, Fumagalli \& Krumholz 2012). A detailed and complete explanation of the Bayesian approach can be found in Krumholz et al. (2015).

Each source in the stellar cluster catalogue that is brighter than -6 mag in the $V$ band, and detected in at least four bands, has been morphologically classified via visual inspection by three independent members of the LEGUS team (Grasha et al. 2015; Adamo et al. 2017). The inspected clusters were divided into four morphological classes: Class 1 contains compact, symmetric, and centrally concentrated clusters. Class 2 includes compact clusters with

\footnotetext{
${ }^{2}$ The magnitude difference between apertures of radius 1 and 3 pixels.
}

a less symmetric light distribution, Class 3 represents less compact and multipeak cluster candidates with asymmetric profiles, and Class 4 consists of unwanted objects like single stars, multiple stars, or background sources. Unclassified objects were labelled as Class 0.

In addition, a machine-learning (ML) approach was tested to morphologically classify the stellar clusters in an automated fashion. A forthcoming paper (Grasha et al., in preparation) will present the ML code that was used for cluster classification in the LEGUS survey and the degree of agreement with human classification. An initial comparison between human and ML classification in M51a was already discussed by Messa et al. (2018).

For our analysis, we use stellar cluster properties estimated with Yggdrasil deterministic models based on the Padova stellar libraries (see Zackrisson et al. 2011 for details) with solar metallicity, the Milky Way extinction curve (Cardelli, Clayton \& Mathis 1989), and the Kroupa (2001) stellar initial mass function. We also selected clusters based on human visual classification for NGC 628, a combination of human and ML classification in NGC 1566, and only ML for M51a. Star clusters classified as Class 4 and Class 0 are excluded from our analysis. Among our target galaxies, there is a total number of 1573,3374 , and 1262 star cluster candidates classified as Classes 1, 2, and 3 in NGC 1566, M51a, and NGC 628, respectively.

A detailed description of the properties of the final cluster catalogues of M51a and NGC 628 and their completeness can be found in Messa et al. (2018) and Adamo et al. (2017).

\subsection{Selection of star clusters of different ages}

In this study, we use the age of star clusters in our galaxy sample as a tool to find a possible age gradient across the spiral arms predicted by the stationary density wave theory. Therefore, we group star clusters into three different cluster samples according to their ages.

The estimated physical properties of star clusters based on the Yggdrasil deterministic models are inaccurate for low-mass clusters (Krumholz et al. 2015). A comparison between the deterministic approach based on Yggdrasil models and the Bayesian approach with SLUG models presented by Krumholz et al. (2015) suggests that the derived cluster properties are uncertain at cluster masses below $5000 \mathrm{M}_{\odot}$. We adopted the same mass cut-off and for NGC 628 and M51a in our analysis. Using the luminosity corresponding to this mass, namely $M_{\mathrm{V}}=-6 \mathrm{mag}\left(m_{\mathrm{V}}=23.4\right.$ and $23.98 \mathrm{mag}$ for NGC 628 and M51a, respectively) results in an age completeness limit of $\leq 200$ Myr. In Adamo et al. (2017) and Messa et al. (2018), 

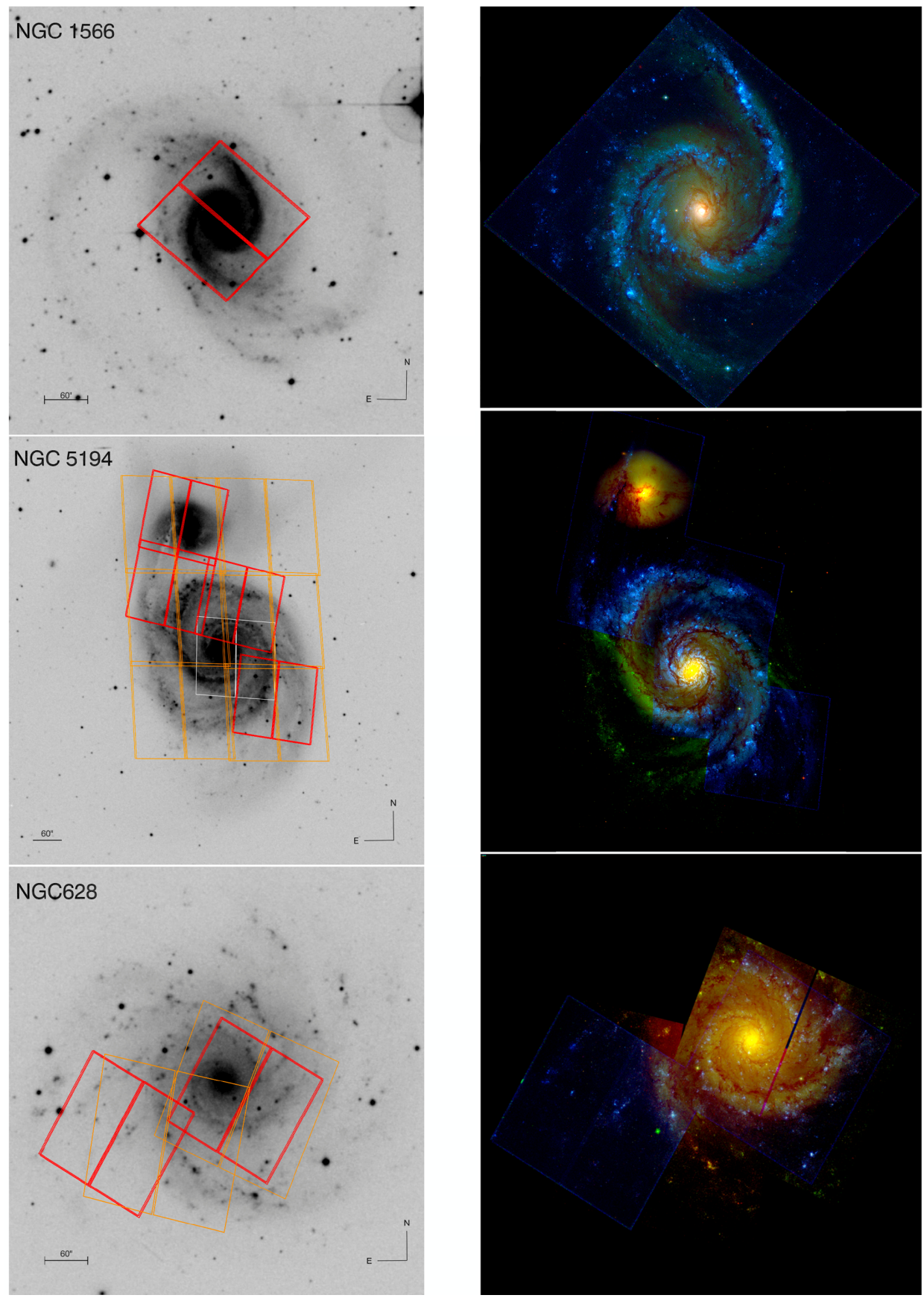

Figure 1. Left: UVIS (red boxes) and ACS (yellow boxes) footprints on DSS images of the galaxies NGC 1566, M51, and NGC 628 (from top to bottom, respectively). The horizontal bar in the lower left corner denotes the length-scale of 60 arcsec. North is up and east to the left. Right: colour composite images for the same galaxies, constructed from LEGUS imaging in the filters $F 275 W$ and $F 336 W$ (blue), $F 438 W$ and $F 555 W$ (green), and $F 814 W$ (red). The central UVIS pointing (white) of M51a was taken from the observations for proposal 13340 (PI: S. Van Dyk).

the magnitude cut at $M_{\mathrm{V}}<-6$ mag is a more conservative limit than the magnitude limit corresponding to 90 per cent of completeness in the recovery of sources. We have tested our results using different mass cuts as well as by removing any constraint on the limiting mass, and we have not observed any significant change in the age distributions of the clusters as a function of azimuthal distances. Thus, the results presented in Sections 5 and 6 are robust against uncertainties in the determination of cluster physical properties.
NGC 1566 is the most distant galaxy within our LEGUS sample. Due to the large distance of this galaxy, the 90 per cent completeness limit $\left(m_{\mathrm{V}}=23.5 \mathrm{mag}\right)$ is significantly brighter than $M_{\mathrm{V}}=-6 \mathrm{mag}$. Therefore, in order to select star clusters in NGC 1566, we used the 90 per cent completeness limit and a mass cut of $5000 \mathrm{M}_{\odot}$ for the cluster ages up to $100 \mathrm{Myr}$ and $10^{4} \mathrm{M}_{\odot}$ for the 100-200 Myr old star clusters (see Fig. 2). Applying these two criteria reduced our cluster samples from 1573 to 1195 clusters for NGC 1566, from 3374 to 1781 clusters for M51a, and from 1262 to 490 for NGC 628. 

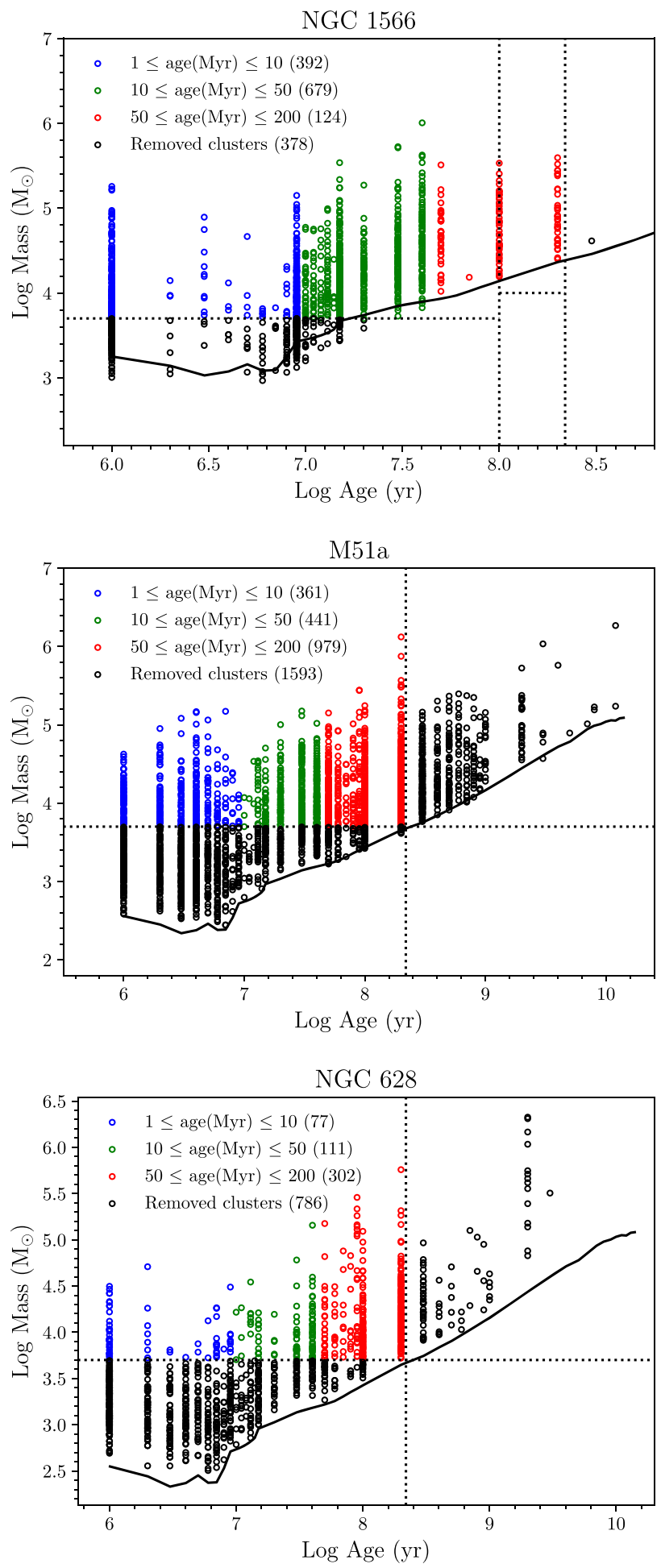

Figure 2. Distribution of ages and masses of the star clusters (classes 1,2, and 3) in NGC 1566, M51a, and NGC 628. The colours represent different age bins: blue (the young sample), green (the intermediate-age sample), red (the old sample), and black (excluded star clusters). The number of clusters in each sample is shown in parentheses. The horizontal dotted lines in NGC 1566 show the applied mass cut of $5000 \mathrm{M}_{\odot}$ up to the age of $100 \mathrm{Myr}$ and $10^{4} \mathrm{M}_{\odot}$ up to the age of $200 \mathrm{Myr}$. The applied mass cut of $5000 \mathrm{M}_{\odot}$ up to the age of 200 Myr in M51a and NGC 628 are also by horizontal dotted lines. The solid black line shows the 90 per cent completeness limit of 23.5 mag in the $V$ band in NGC 1566 and the magnitude cut of $M_{\mathrm{V}}=-6$ mag in M51a, and NGC 628, respectively.
Then, we selected three cluster samples of different ages for each galaxy as follows:

- 'Young' star clusters: age (Myr) $<10$

- 'Intermediate-age' star clusters: $10 \leq$ age (Myr) $<50$

- 'Old' star clusters: $50 \leq$ age (Myr) $\leq 200$

The number of star clusters in the 'young', 'intermediate-age', and 'old' samples is shown in Table 2.

Fig. 2 displays the age-mass diagram of star clusters in NGC 1566, M51a, and NGC 628. The young, the intermediate-age, and the old star cluster samples are shown in blue, green, and red colours, respectively. The excluded star clusters (due to the mass cut) are shown in black. The horizontal and vertical dotted lines show the applied mass cut of $5000 \mathrm{M}_{\odot}$ and its corresponding completeness limit at a stellar age of $200 \mathrm{Myr}$, respectively.

\section{SPATIAL DISTRIBUTION AND CLUSTERING OF STAR CLUSTERS}

In Fig. 3, we plot the spatial distribution of star clusters of different ages in the galaxies NGC 1566, M51a, and NGC 628. The young, intermediate-age, and old stellar cluster samples are shown in blue, green, and red, respectively. In general, we observe a similar trend in our target galaxies: first, the young and the intermediate-age star clusters mostly populate the spiral arms rather than the interarm regions. This is particularly evident for NGC 1566 and M51a, which show strong and clear spiral structures in young and intermediateage star clusters. Second, the old star clusters are less clustered and more widely spread compared to the young and intermediate-age star cluster samples.

Our findings are similar to other literature results on the spatial distribution of star clusters of different ages: Dobbs et al. (2017), using LEGUS HST data found that in NGC 1566 the $100 \mathrm{Myr}$ old star clusters clearly trace the spiral arms, while in NGC 628 star clusters older than 10 Myr show only weak spiral structures. Chandar et al. (2017), using other HST data observed that M51a shows weak spiral structure in older star clusters (>100 Myr).

Clustering of star clusters has been observationally investigated for a number of local star-forming galaxies (e.g. Efremov 1995; Efremov \& Elmegreen 1998). In a detailed study of clustering of the young stellar population in NGC 6503 based on the LEGUS observations, Gouliermis et al. (2015) found that younger stars were more clustered compared to the older ones. Grasha et al. (2015) investigated the spatial distribution of the star clusters in NGC 628 from the LEGUS sample. Their findings confirmed that the degree of the clustering increases with decreasing age. More recently, Grasha et al. (2017) studied the hierarchical clustering of young star clusters in a sample of six LEGUS galaxies. Their results suggested that the youngest star clusters are strongly clustered and the degree of clustering quickly drops for clusters older than $20 \mathrm{Myr}$ and the galactic shear appears to drive the largest sizes of the hierarchy in each galaxy (Grasha et al. 2017).

Adopting a similar approach as Grasha et al. (2015), we use the two-point correlation function to test whether or not the clustering distribution of the clusters in our selected age bins shows the expected age dependence. The two-point correlation function $\omega(\theta)$ is a powerful statistical tool for quantifying the probability of finding two clusters with an angular separation $\theta$ against a random, non-clustered distribution (Peebles 1980). Here, we use the LandySzalay (Landy \& Szalay 1993) estimator, which has little sensitivity 
Table 2. The number of star clusters in the 'young', 'intermediate-age', and 'old' samples in our target galaxies.

\begin{tabular}{lccc}
\hline galaxy & Age $($ Myr $)<10$ & $10 \leq$ age $($ Myr $)<50$ & $50 \leq$ age $($ Myr $) \leq 200$ \\
\hline NGC 1566 & 392 & 679 & 124 \\
M51a & 361 & 441 & 979 \\
NGC 628 & 77 & 111 & 302 \\
\hline
\end{tabular}
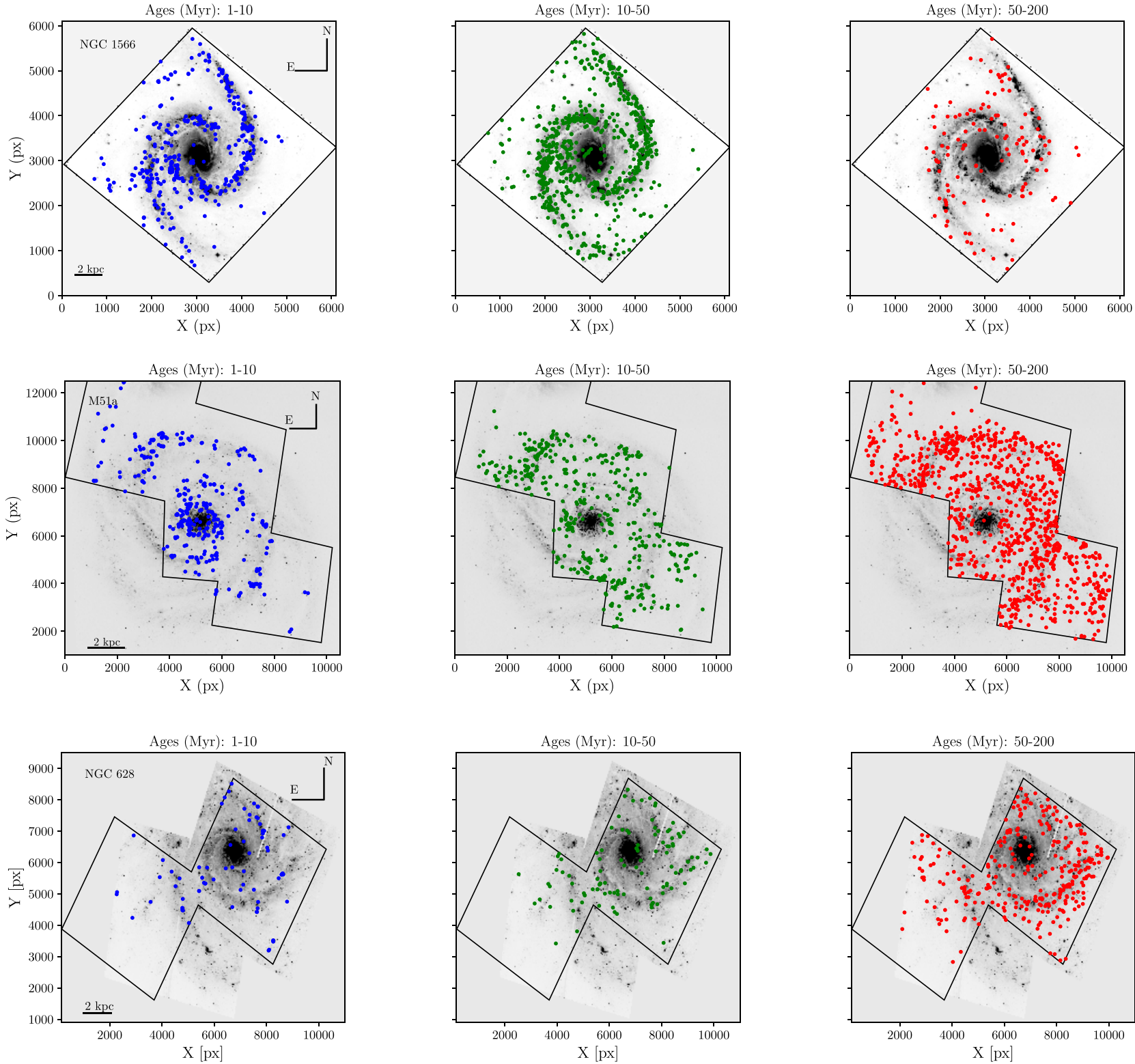

Figure 3. The spatial distribution of star clusters of different age in the galaxies NGC 1566, M51a, and NGC 628 (from top to bottom) superimposed on the $B$-band images. The blue, green, and red circles show the young (age $(\mathrm{Myr})<10)$, intermediate-age $(10 \leq$ age $(\mathrm{Myr})<50)$, and old star clusters $(50 \leq \mathrm{age}$ $(\mathrm{Myr}) \leq 200)$, respectively. The black outlines show the UVIS footprints. The horizontal bar in the lower left corner denotes the length of $2 \mathrm{kpc}$. North is up and east to the left.

to the presence of edges and masks in the data:

$\omega(\theta)=\frac{r(r-1)}{n(n-1)} \frac{\mathrm{DD}}{\mathrm{RR}}-\frac{(r-1)}{n} \frac{\mathrm{DR}}{\mathrm{RR}}+1$,

where $n$ and $r$ are the total number of data and random points, respectively. DD, RR, and DR are the total numbers of data-data, random-random, and data-random pair counts with a separation $\theta \pm \mathrm{d} \theta$, respectively. We construct a random dis- tribution of star clusters that has the same sky coverage and masked regions (e.g. the ACS chip gap) as the images of each galaxy.

Fig. 4 displays the two-point correlation function for the star clusters in different age bins as defined for our galaxy samples. The blue, green, and red colours represent the young, intermediate-age, and old star cluster samples in each galaxy, respectively. The error 

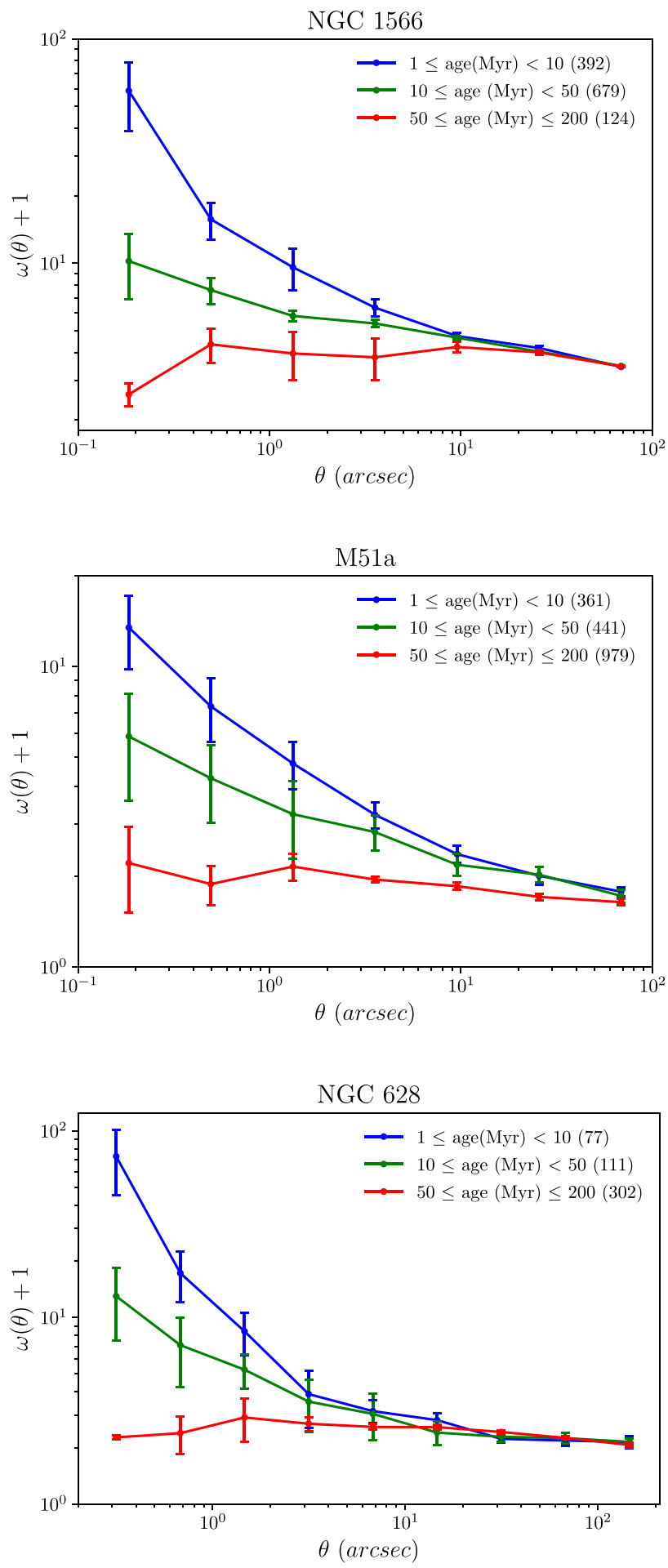

Figure 4. Two-point correlation function for the star cluster samples of different ages as a function of angular distance (arcseconds) in NGC 1566, M51a, and NGC 628. The young, intermediate-age, and old star cluster samples are shown in blue, green, and red, respectively. The error bars were computed based on a bootstrapping method. The number of star clusters in each age bin are listed in parentheses. bars on the two-point correlation function were estimated using a bootstrapping method with 1000 bootstrap resamples.

The general distribution of the star cluster samples in the target galaxies shows a similar trend: independent of the presence of spiral arms, young clusters show hierarchical structure, whilst the old star clusters show a non-clustered, smooth distribution.

\section{ARE THE SPIRAL ARMS STATIC DENSITY WAVES?}

As discussed in Section 1, the stationary density wave theory foresees that the age of stellar clusters inside the corotation radius increases with increasing distance from the spiral arms. In other words, we expect to find a shift in the location of stellar clusters with different ages.

In order to test whether the distribution of star clusters of different ages in our target galaxies agrees with the expectations from the stationary density wave theory, we need to quantify the azimuthal offset between star clusters of different ages.

\subsection{Spiral arm ridge lines definition}

First of all, we need to locate the spiral arms of our galaxy sample. We wish to define a specific location in each spiral arm so we can measure the relative positions of the star clusters in a uniform way. We use the dust lanes for this purpose because they are narrow and well defined on optical images.

As gas flows into the potential minima of a density wave, it gets compressed and forms dark dust lanes in the inner part of the spiral arms, where star formation is then likely to occur (Roberts 1969). We have used the $B$-band images for this purpose, since most of the emission is due to young $\mathrm{OB}$ stars and dark obscuring dust lanes can be better identified in this band.

To better define the average positions of the dust lanes, we used a Gaussian kernel (with a 10 pixels sigma) to smooth the images, reduce the noise, and enhance the spiral structure. In the smoothed images, the dust lanes are clearly visible as dark ridges inside the bright spiral arms. We defined these dark spiral arm ridge lines manually. For the remainder of this paper, we refer to the southern and northern arms as 'Arm 1' and 'Arm 2', respectively. Fig. 5 presents the defined spiral arm ridge lines (red lines) overplotted on the smoothed $B$-band images of NGC 1566, M51a, and NGC 628

\subsection{Measuring azimuthal offset}

Knowing the position of star clusters and spiral arm ridge lines in our target galaxies allowed us to measure the azimuthal distance of a star cluster from its closest spiral arm, assuming that it rotates on a circular orbit.

We limited our analysis to the star clusters located in the disc where spiral arms exist. The disc of a galaxy can be defined by its rotation curve. The rotational velocity increases when moving outwards from the central bulge-dominated part and becomes flat in the disc-dominated part of the galaxy. We derived a radius of $2 \mathrm{kpc}$ for the bulge-dominated part of our galaxies using the rotation curves of Korchagin et al. (2000) for NGC 1566, Sofue (1996, 1997) for M51a, and Combes \& Becquaert (1997) for NGC 628. Furthermore, we limited our analysis to star clusters located inside the corotation radius. If stationary density waves are the dominant 

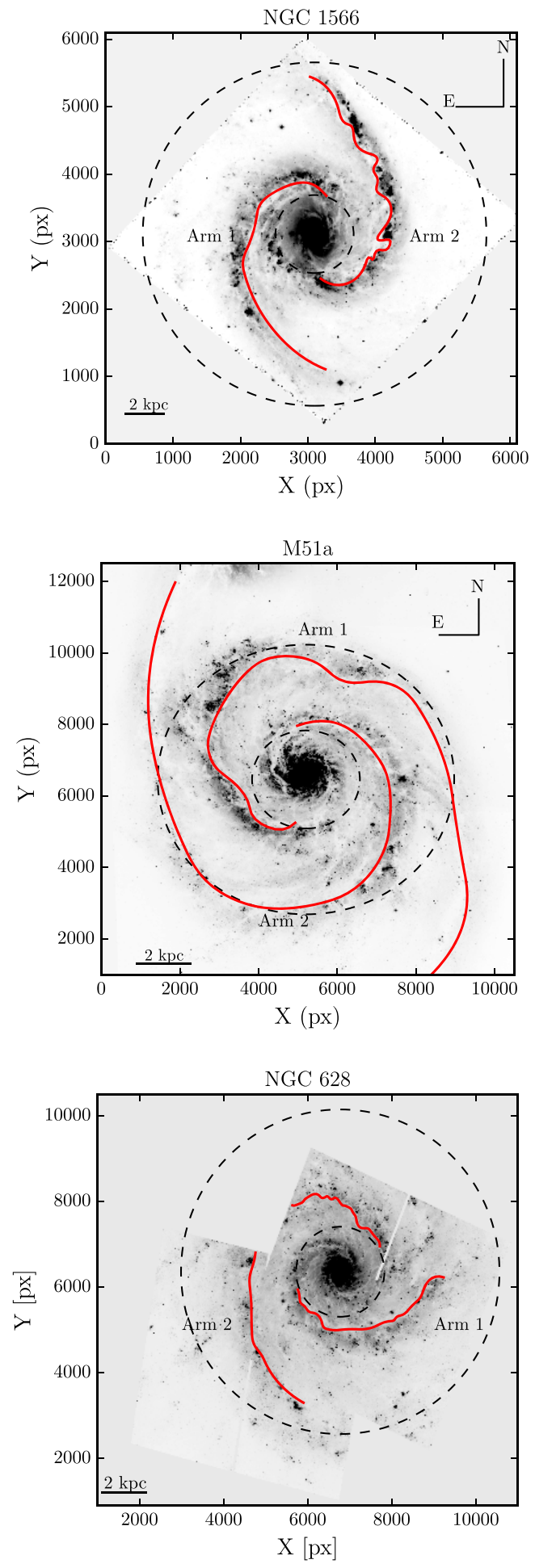

Figure 5. The location of spiral arm ridge lines is shown by red lines overplotted on the smoothed $B$-band images of NGC 1566, M51a, and NGC 628. We refer to the southern and northern arms as 'Arm 1' and 'Arm 2', respectively. The two black dashed circles in each panel mark the onset of the bulge and the location of the corotation radius of the galaxies. The horizontal bar in the lower left corner denotes a length-scale of $2 \mathrm{kpc}$. North is up and east to the left. mechanism driving star formation in spiral galaxies we expect to find an age gradient from younger to older clusters inside the corotation radius. The bulge-dominated region and corotation radius of each galaxy are shown in Fig. 5. The adopted corotation radii of the galaxies are listed in Table 1 .

Fig. 6 (left-hand panels) shows the normalized distribution of the azimuthal distance of star clusters in the three age bins from their closest spiral arm ridge line in NGC 1566, M51a, and NGC 628. The error bars in each sample were calculated by dividing the square root of the number of clusters in each bin by the total number of clusters. We note that an azimuthal distance of zero degrees shows the location of the spiral arm ridge lines and not the centre of the arms. Positive (negative) azimuthal distributions indicate that a cluster is located in front of (behind) the spiral arm ridge lines. Blue, green, and red colours represent the young, intermediate-age, and old star cluster samples, respectively.

Fig. 6 (right-hand panels) shows the cumulative distribution function of star clusters as a function of the azimuthal distance. In order to test whether the samples come from the same distribution, we used a two-sample Kolmogorov-Smirnov test (hereafter $\mathrm{K}-\mathrm{S}$ test). Since we aim at finding the age gradient in front of the spiral arms, the $\mathrm{K}-\mathrm{S}$ test was only calculated for star clusters with positive azimuthal distances. The probability that two samples are drawn from the same distribution ( $p$-values) and the maximum difference between pairs of cumulative distributions $(D)$ are listed in Table 3.

In the case of NGC 1566 (Fig. 6, top), we see that the young and intermediate-age star cluster samples are peaking close to the location of the spiral arm ridge lines (azimuthal distance of 0 $5 \mathrm{deg}$ ), while the old sample peaks further away from the ridge lines (azimuthal distances of 5-10 deg). The derived $p$-values are lower than the test's significance level (0.05) of the null hypothesis, i.e. that the two samples are drawn from the same distribution. As a consequence, our three star cluster samples are unlikely to be drawn from the same population. A clear age gradient across the spiral arms can be observed in NGC 1566, which is in agreement with the expectation from stationary density wave theory. The existence of such a pattern supports the picture of an age sequence in the model of a grand-design spiral galaxy and a barred galaxy suggested by Dobbs \& Pringle (2010) and Gouliermis et al. (2017).

No obvious age gradient from younger to older is seen in the azimuthal distributions of the star cluster samples in M51a (Fig. 6, middle). What is remarkable here is that the older star clusters are located closer to the spiral arm ridge lines than the young and intermediate-age star clusters. The $\mathrm{K}-\mathrm{S}$ test indicates that the probability that the young star cluster sample is drawn from the same distribution as the intermediate-age and old star cluster samples is more than 10 percent. The derived $p$-value for the intermediateage and old cluster samples is lower than the significance level of the $\mathrm{K}-\mathrm{S}$ test and rejects the null hypothesis that the two samples are drawn from the same distribution. The lack of an age pattern is consistent with the observed age trend for an interacting galaxy, modelled based on M51a, suggested by Dobbs \& Pringle (2010). Our result is compatible with a number of observational studies have found no indication for the expected spatial offset from the stationary density wave theory in M51a (Scheepmaker et al. 2009; Kaleida \& Scowen 2010; Foyle et al. 2011; Schinnerer et al. 2017).

There is no evident trend in the azimuthal distribution of star clusters in NGC 628 (Fig. 6, bottom). The majority of the young star clusters tends to be located further away from the ridge lines (azimuthal distance of 20-25 deg). The calculated $p$-values from the 

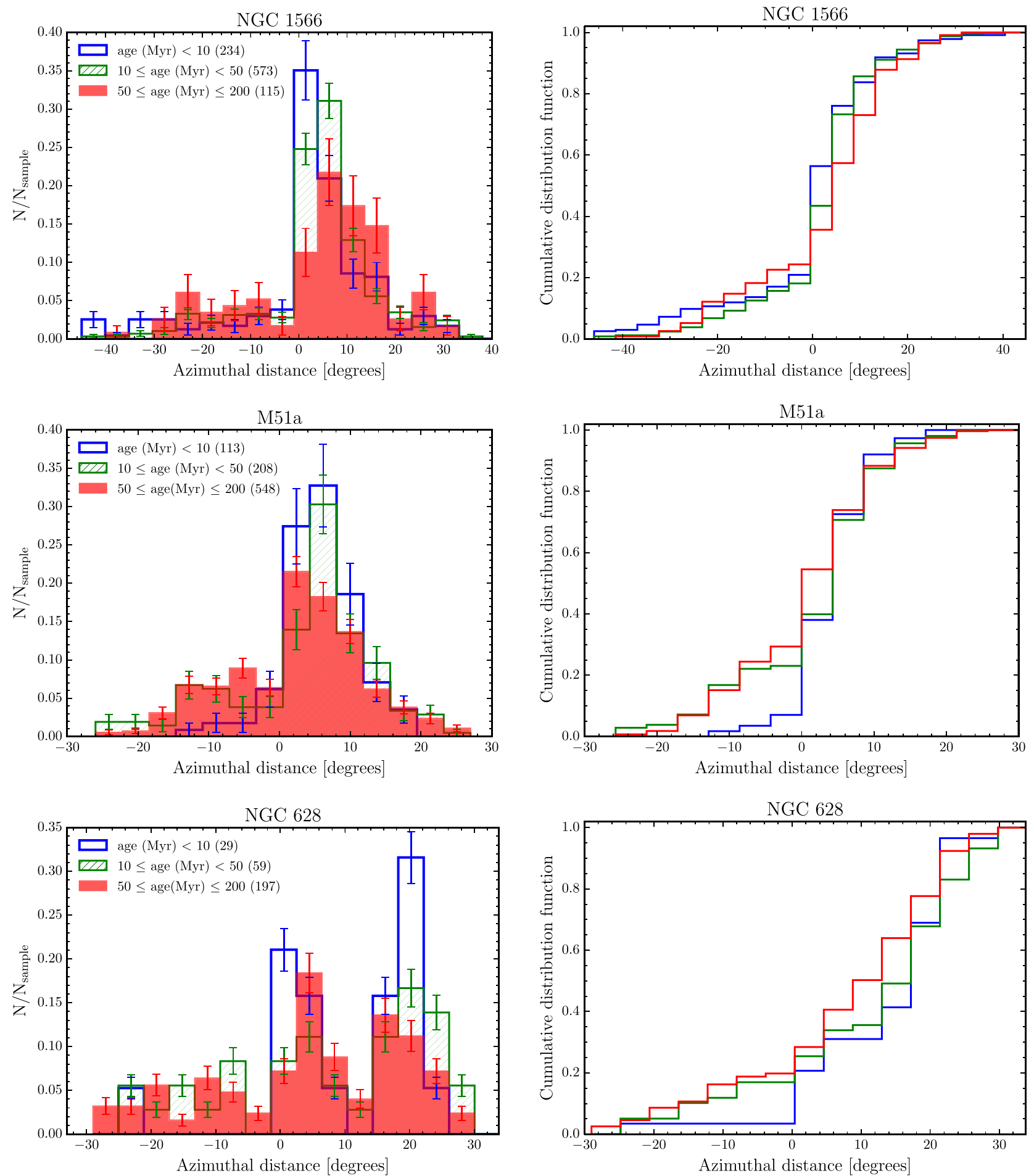

Figure 6. The normalized distribution of azimuthal distance (in degrees) of the star cluster samples from their closest spiral arm (left-hand panels) and the cumulative distribution function of star clusters of different ages as a function of the azimuthal distances (in degrees) in NGC 1566, M51a, and NGC 628. The young ( $<10 \mathrm{Myr}$ ), intermediate-age (10-50 Myr), and old star cluster samples (50-200 Myr) are shown in blue, green, and red, respectively. The number of star clusters located in the disc and inside the corotation radius of each galaxy is listed in parentheses.

$\mathrm{K}-\mathrm{S}$ test are larger than 0.05 , which suggests weak evidence against the null hypothesis. As a result, the three young, intermediate-age, and old star cluster samples are drawn from the same distribution. The absence of an age gradient across the spiral arms in NGC 628 is consistent with a simulated multiple arm spiral galaxy by Grand et al. (2012).

\section{THE ORIGIN OF TWO SPIRAL ARMS}

An observational study by Egusa et al. (2017), based on measuring azimuthal offsets between the stellar mass (from optical and nearinfrared data) and gas mass distributions (from $\mathrm{CO}$ and $\mathrm{HI}$ data) in two spiral arms of M51a, suggest that the origin of these spiral arms 
Table 3. The maximum difference between pairs of cumulative distributions $(D)$ of azimuthal distance of star clusters and the probability that two samples are drawn from the same distribution ( $p$-values) of the two sample K-S test in NGC 1566, M51a, and NGC 628.

\begin{tabular}{|c|c|c|c|c|c|c|}
\hline \multirow[t]{2}{*}{ galaxy } & \multicolumn{2}{|c|}{ Young and intermediate age } & \multicolumn{2}{|c|}{ Young and old } & \multicolumn{2}{|c|}{ Intermediate age and old } \\
\hline & $D$ & $p$-value & $D$ & $p$-value & $D$ & $p$-value \\
\hline NGC 1566 & 0.15 & $3.78 \times 10^{-3}$ & 0.31 & $2.88 \times 10^{-5}$ & 0.26 & $6.19 \times 10^{-5}$ \\
\hline NGC 628 & 0.21 & 0.49 & 0.17 & 0.47 & 0.19 & 0.10 \\
\hline
\end{tabular}

differs. One spiral arm obeys the stationary density wave theory, while the other does not.

In another recent study of M51a, Chandar et al. (2017) quantified the spatial distribution of star clusters with different ages relative to different segments of the two spiral arms of M51a traced in the $3.6 \mu \mathrm{m}$ image. They observed a similar trend for the western and eastern arms: the youngest star clusters $(<6 \mathrm{Myr})$ are found near the spiral arm segments, and the older clusters (100-400 Myr) show an extended distribution.

In this section, we test whether measuring the azimuthal offset of star cluster samples from each spiral arm individually leads to different results. We assume that a star cluster whose distance from Arm 1 is smaller than its distance from Arm 2 belongs to Arm 1 and vice versa.

Fig. 7 shows the normalized distribution of ages of star clusters associated with Arm 1 (shown in red) and Arm 2 (shown in blue) in each of the galaxies. No significant differences between the age distribution of star clusters belonging to the two spiral arms in our target galaxies can be observed. Also, the $\mathrm{K}-$ $\mathrm{S}$ test indicates that the age distributions of star clusters relative to Arm 1 and Arm 2 in each galaxy are drawn from the same population.

In Fig. 8, we compare the normalized azimuthal distribution of the three young, intermediate-age, and old star cluster samples relative to Arm 1 (left-hand panels) and Arm 2 (right-hand panels) in our target galaxies. As before, our analysis was limited to the star clusters positioned in the disc and inside the corotation radius of our target galaxies.

The upper panels of Fig. 8 exhibit a noticeable age gradient across both spiral arms of NGC 1566. The young star clusters are highly concentrated towards the location of Arm 1 and Arm 2, while the older ones are peaking further away from the two spiral arms.

The second row panels of Fig. 8 show the azimuthal distance of star cluster samples across the two arms of M51a. This galaxy displays an offset in the location of young and old star clusters across Arm 1. The young star clusters culminate close to Arm 1 (at azimuthal distances of 2-6 deg), while the old ones are positioned further away (at azimuthal distances of 6-10 deg). Even though M51a shows an age gradient across the Arm 1 at first glance, the $\mathrm{K}-\mathrm{S}$ test does not imply significant differences between the young and old star cluster samples (all derived $p$-values are larger than the test's significance level). We do not observe any shift in the azimuthal distribution of the star cluster samples across Arm 2 in M51a.

In the case of NGC 628, no obvious age gradient across Arm 1 and Arm 2 is observed (the lower panels of Fig. 8). It is important to note that our results are inconclusive for the young star clusters associated with Arm 2 due to the small number statistics. Hence, we also explored the change in the azimuthal distribution of the star clusters by including clusters with masses $<5000 \mathrm{M}_{\odot}$ and
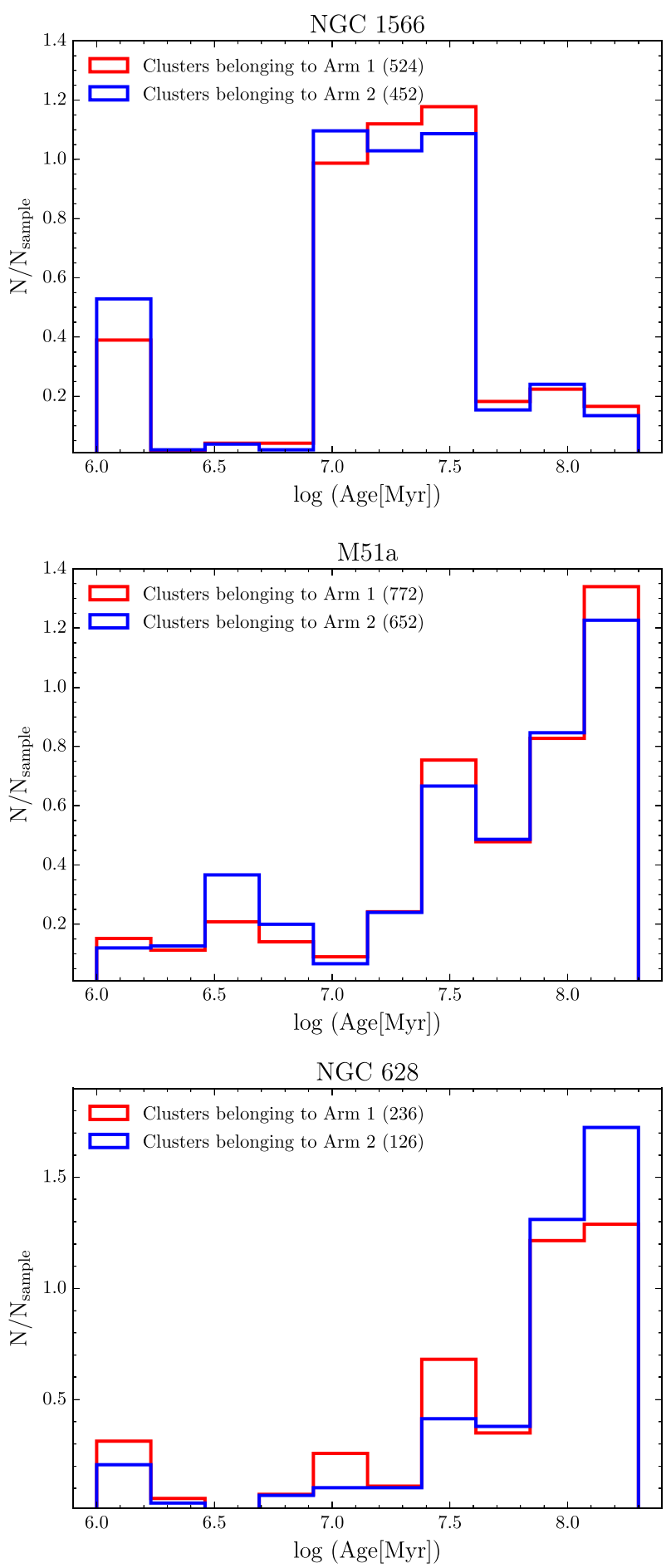

Figure 7. The distribution of the age of star clusters associated with Arm 1 (red) and Arm 2 (blue) in NGC 1566, M51a, and NGC 628. The number of star clusters relative to the Arm 1 and Arm 2 is listed in parantheses. 

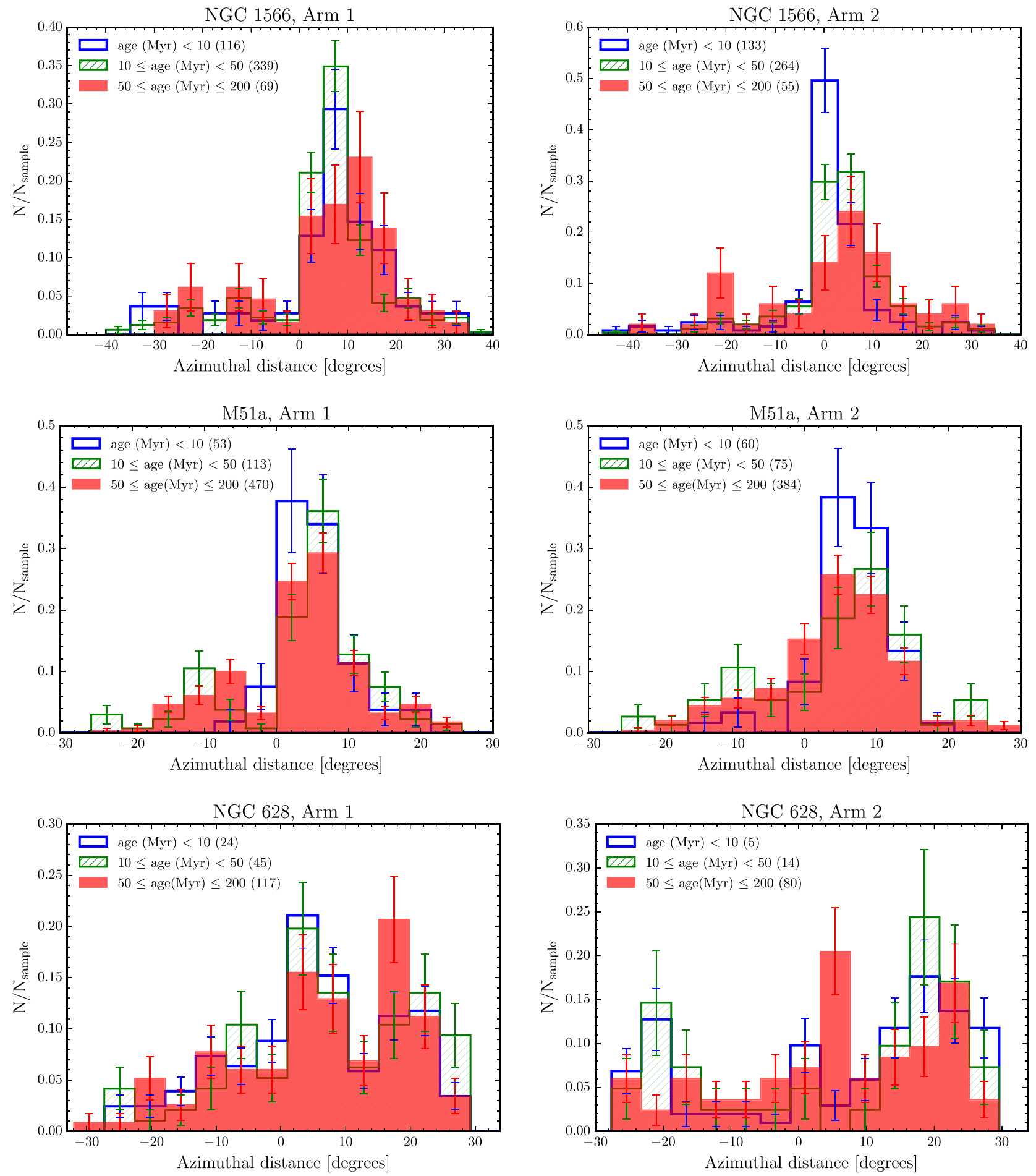

Figure 8. The normalized distribution of azimuthal distance (in degrees) of the star cluster samples belonging to Arm 1 (left-hand panels) and Arm 2 (right-hand panels) in NGC 1566, M51a, and NGC 628. Blue, green, and red colours present the young (<10 Myr), intermediate-age (10-50 Myr), and old star cluster samples (50-200 Myr), respectively. The number of star clusters corresponding to Arm 1 and Arm 2 is listed in parantheses. The error bars in each sample were calculated by dividing the square root of the number of clusters in each bin by the total number of clusters.

ages > 200 Myr. The observed differences are not significant and the general trend is the same as before.

Thus, measuring the azimuthal distance of the star clusters from the two individual spiral arms in each galaxy suggests that the two spiral arms of our target galaxies may have the same physical origin.

\section{COMPARISON WITH THE NON-LEGUS CLUSTER CATALOGUE OF M51}

In this section, we use the Chandar et al. (2016) catalogue (hereafter CH16 catalogue) to measure the azimuthal offsets of star clusters with different ages in M51a and to compare the results with our 


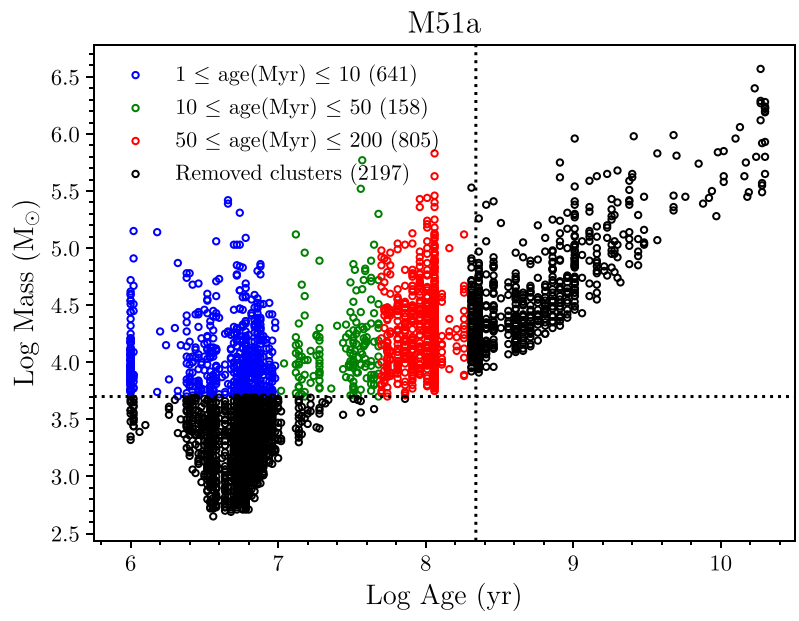

Figure 9. The distribution of ages and masses of the 3816 star clusters in M51a, based on the CH16 catalogue. The young ( $<10 \mathrm{Myr})$, intermediateage (10-50 Myr), and old (50-200 Myr) star clusters are shown in blue, green, and red, respectively. The black points indicate excluded star clusters due to the applied mass cut and the imposed completeness limit. The number of clusters in each sample is listed in parentheses. The horizontal and vertical dotted lines show the applied mass cut of $5000 \mathrm{M}_{\odot}$ and the corresponding detection completeness limit of $200 \mathrm{Myr}$, respectively.

analysis based on the LEGUS catalogue. We caution that the southeastern region of M51a is not covered by the LEGUS observations. We also investigated whether our results are biased due to the absence of star clusters from that region.

Chandar et al. (2016) provided a catalogue of 3816 star clusters in M51a based on HST ACS/WFC2 images obtained the equivalents of $U B V I$ and $\mathrm{H} \alpha$ filters. Messa et al. (2018) compared the age distributions of star clusters in common between the LEGUS and CH16 catalogue. They observed that a large number of young star clusters (age < $10 \mathrm{Myr}$ ) in Chandar et al. (2016) have a broad age range (age: 1-100 Myr) in the LEGUS catalogue. They argued that the discrepancies in the estimated ages are due to the use of different filter combinations.

In Fig. 9, we show the distribution of ages and masses of star clusters in M51a from the CH16 catalogue. In order to be able to compare our results, we considered a mass-limited sample with masses >> $5000 \mathrm{M}_{\odot}$ and ages < $200 \mathrm{Myr}$ and selected the same age bins as before: the young $(<10 \mathrm{Myr})$, intermediate-age (10 $50 \mathrm{Myr}$ ), and old star cluster samples (50-200 Myr).

In Fig. 10, we plot the spatial distribution of the young, intermediate-age, and old star clusters based on the $\mathrm{CH} 16$ catalogue in M51a. As we can see, M51a displays a very clear and strong spiral pattern in the young star clusters. The intermediateage star clusters tend to be located along the spiral arms, while the old ones are more scattered and populate the interarm regions. Recently, Chandar et al. (2017) using the CH16 catalogue found that the youngest star clusters $(<6 \mathrm{Myr})$ are concentrated in the spiral arms (defined based on $3.6 \mu \mathrm{m}$ observations). The older star clusters (6-100 Myr) are also found close to the spiral arms but they are more dispersed, and the spiral structure is not clearly recognizable in older star clusters ( $>400 \mathrm{Myr}$ ).

In order to quantify the possible spatial offset in the location of the three young, intermediate-age, and old star cluster samples from the $\mathrm{CH} 16$ catalogue across the spiral arms, we computed the normalized azimuthal distribution and corresponding cumulative distribution function of the star cluster samples in Fig. 11. We applied our analysis only to the star clusters positioned in the disc and inside the corotation radius of M51a (2.0-5.5 kpc). Our result demonstrates that the three young, intermediate-age, and old star cluster samples peak at an azimuthal distance of $6 \mathrm{deg}$ from the location of the spiral arms. We observe no obvious offsets between the azimuthal distances of the three star cluster age samples in M51a. Chandar et al. (2017), using the same cluster catalogue, quantified the azimuthal offset of molecular gas (from PAWS and HERACLES) and young (<10 Myr) and intermediate-age (100$400 \mathrm{Myr})$ star clusters in the inner $(2-2.5 \mathrm{kpc})$ and outer $(5-5.5 \mathrm{kpc})$ annuli of the spiral arms. They found that in the inner annuli the young star clusters show an offset of $1 \mathrm{kpc}$ from the molecular gas, while there is no offset between the molecular gas and young and old star clusters in the outer portion of the spiral arms.

Adopting the $\mathrm{CH} 16$ catalogue, we found that there is no noticeable age gradient across the spiral arms of M51a, which is in agreement with our finding based on the LEGUS star cluster catalogue.

\section{DISCUSSION AND CONCLUSIONS}

The stationary density wave theory predicts that the age of star clusters increases with increasing distance away from the spiral arms. Therefore, a simple picture of the stationary density wave theory leads to a clear age gradient across the spiral arms. In this study, we are testing the theory that spiral arms are static features with constant pattern speed. For this purpose, we use the age and position of star clusters relative to the spiral arms.

We use high-resolution imaging observations obtained by the LEGUS survey (Calzetti et al. 2015) for three face-on LEGUS spiral galaxies, NGC 1566, M51a, and NGC 628. We have measured the azimuthal distance of the LEGUS star clusters from their closest spiral arm to quantify the possible spatial offset in the location of star clusters of different ages ( $<10,10-50$, and 50-200 Myr) across the spiral arms. We found that the nature of spiral arms in our target galaxies is not unique. The main results are summarized as follows:

(i) Our detailed analysis of the azimuthal distribution of star clusters indicates that there is an age sequence across spiral arms in NGC 1566. NGC 1566 shows a strong bar and bisymmetric arms typical of a massive self-gravitating disc (D'Onghia 2015). We speculate that when discs are very self-gravitating the bar and the two-armed features dominate a large part of the galaxy, producing an almost constant pattern speed. The observed trend is also in agreement with what was found by Dobbs \& Pringle (2010) in simulations of a grand design and a barred spiral galaxy.

(ii) We find no age gradient across the spiral arms of M51a. This galaxy shows less strong arms and a weaker bar and hence a less self-gravitating disc. The absence of an age sequence in M51a indicates that the grand-design structures of this galaxy are not the result of a steady-state density wave, with a fixed pattern speed and shape, as in the early analytical models. More likely, the spiral is a density wave that is still changing its shape and amplitude with time in reaction to the recent tidal perturbations. A possible mechanism to explain the formation and presence of grand-design structures in spiral galaxies is an interaction with a nearby companion (Toomre \& Toomre 1972; Kormendy \& Norman 1979; Bottema 2003). Since such an interaction is obviously occurring in M51a, tidal interactions could be the dominant mechanism for driving its spiral patterns. Dobbs \& Pringle (2010) simulated M51a with an interacting companion (M51b), and observed no age gradient across the tidally induced grand-design spirals arms. Our findings are con- 

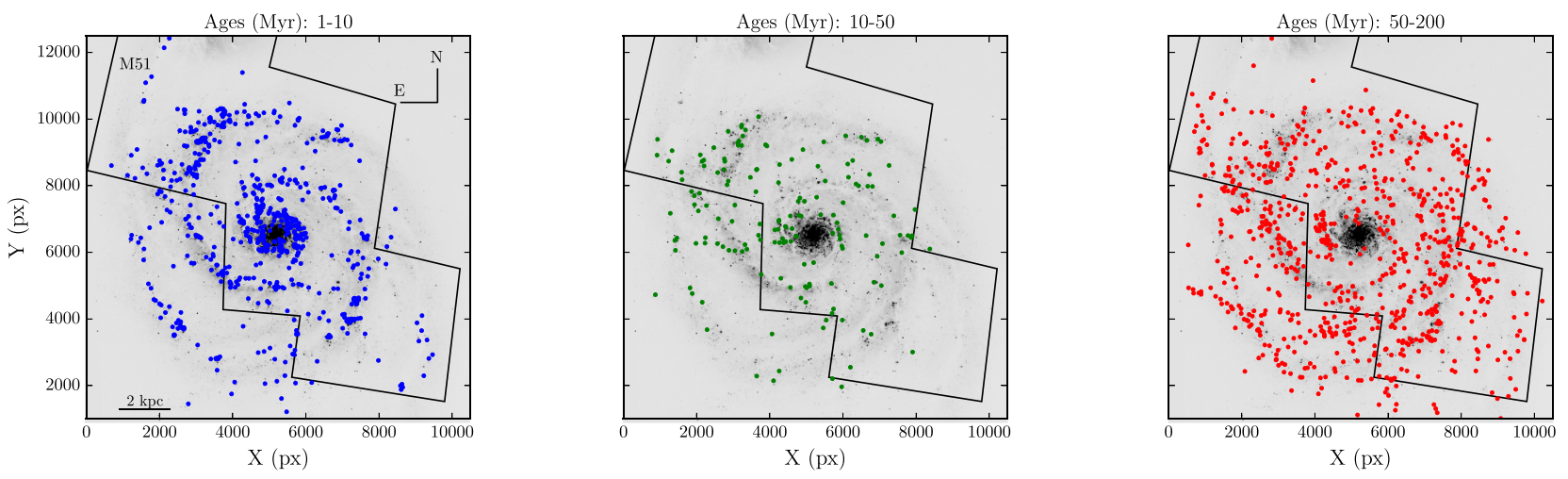

Figure 10. The spatial distribution of the young (blue), intermediate-age (green), and old (red) star clusters in M51a taken from the CH16 catalogue. The black outlines show the area covered by the LEGUS observations. The horizontal bar indicates the length of $2 \mathrm{kpc}$, corresponding to 54 arcsec. North is up and east to the left.
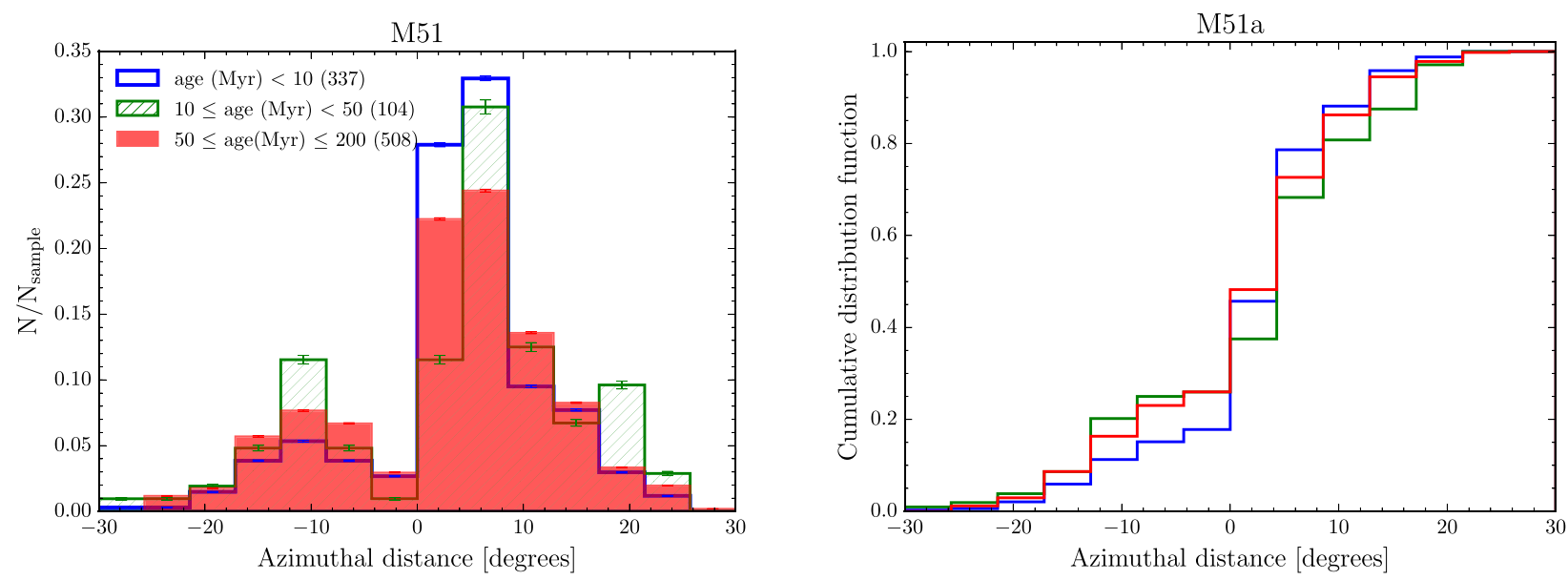

Figure 11. The normalized azimuthal distribution (left) and the cumulative distribution function as a function of the azimuthal distance (right) of three star cluster samples in M51a, based on the CH16 catalogue. The young, intermediate-age, and old star clusters are shown in blue, green, and red, respectively. The number of star clusters in each age bin (located in the disc and inside the corotation radius of M51a) is listed in parentheses. The error bars in each sample were calculated by dividing the square root of the number of clusters in each bin by the total number of clusters.

sistent with the results of several other observational studies, which did not find age gradients as expected from the spiral density wave theory in M51a (Scheepmaker et al. 2009; Kaleida \& Scowen 2010; Foyle et al. 2011; Schinnerer et al. 2017).

(iii) NGC 628 is a multiple-arm spiral galaxy with weak spiral arms consistent with a pattern speed decreasing with radius and multiple corotation radii. In this case, we find no significant offset among the azimuthal distributions of star clusters with different ages, which is consistent with the swing amplification theory. The lack of such an age offset is in agreement with an earlier analysis of NGC 628 (Foyle et al. 2011), and consistent with the spatial distribution of star clusters with different ages in the simulated multiple-arm spiral galaxy by Grand et al. (2012).

\section{ACKNOWLEDGEMENTS}

This work is based on observations made with the NASA/ESA HST, obtained at the Space Telescope Science Institute, which is operated by the Association of Universities for Research in Astronomy, Inc., under NASA contract NAS 5-26555. These observations are associated with program 13364. Support for Program 13364 was provided by NASA through a grant from the Space Telescope Science Institute.
This research has made use of the NED, which is operated by the Jet Propulsion Laboratory, California Institute of Technology, under contract with the National Aeronautics and Space Administration.

AA acknowledges the support of the Swedish Research Council (Vetenskapsrådet) and the Swedish National Space Board. DAG kindly acknowledges financial support by the German Research Foundation (DFG) through programme GO 1659/3-2.

\section{REFERENCES}

Adamo A. et al., 2017, ApJ, 841, 131

Agüero E. L., Díaz R. J., Bajaja E., 2004, A\&A, 414, 453

Athanassoula E., 1992, MNRAS, 259, 345

Bertin E., Arnouts S., 1996, A\&AS, 117, 393

Bothwell M. S., Kennicutt R. C., Lee J. C., 2009, MNRAS, 400, 154

Bottema R., 2003, MNRAS, 344, 358

Calzetti D. et al., 2015, AJ, 149, 51

Cardelli J. A., Clayton G. C., Mathis J. S., 1989, ApJ, 345, 245

Chandar R., Whitmore B. C., Dinino D., Kennicutt R. C., Chien L.-H., Schinnerer E., Meidt S., 2016, ApJ, 824, 71

Chandar R. et al., 2017, ApJ, 845, 78

Combes F., Becquaert J.-F., 1997, A\&A, 326, 554

Combes F. et al., 2014, A\&A, 565, A97

Contopoulos G., Papayannopoulos T., 1980, A\&A, 92, 33 
Cornett R. H. et al., 1994, ApJ, 426, 553

D'Onghia E., 2015, ApJ, 808, L8

D’Onghia E., Vogelsberger M., Hernquist L., 2013, ApJ, 766, 34

D’Onghia E., Madau P., Vera-Ciro C., Quillen A., Hernquist L., 2016, ApJ, 823,4

da Silva R. L., Fumagalli M., Krumholz M., 2012, ApJ, 745, 145

Dobbs C., Baba J., 2014, PASA, 31, e035

Dobbs C. L., Pringle J. E., 2010, MNRAS, 409, 396

Dobbs C. L. et al., 2017, MNRAS, 464, 3580

Efremov Y. N., 1995, AJ, 110, 2757

Efremov Y. N., Elmegreen B. G., 1998, MNRAS, 299, 588

Egusa F., Kohno K., Sofue Y., Nakanishi H., Komugi S., 2009, ApJ, 697, 1870

Egusa F., Mentuch Cooper E., Koda J., Baba J., 2017, MNRAS, 465, 460

Elmegreen B. G., 2011, ApJ, 737, 10

Elmegreen B. G., Elmegreen D. M., 1986, ApJ, 311, 554

Elmegreen D. M., Elmegreen B. G., 1987, ApJ, 314, 3

Elmegreen D. M. et al., 2011, ApJ, 737, 32

Elmegreen D. M. et al., 2014, ApJ, 780, 32

Ferreras I., Cropper M., Kawata D., Page M., Hoversten E. A., 2012, MNRAS, 424, 1636

Foyle K., Rix H.-W., Dobbs C. L., Leroy A. K., Walter F., 2011, ApJ, 735, 101

Gauthier J.-R., Dubinski J., Widrow L. M., 2006, ApJ, 653, 1180

Goldreich P., Lynden-Bell D., 1965, MNRAS, 130, 125

Gouliermis D. A. et al., 2015, MNRAS, 452, 3508

Gouliermis D. A. et al., 2017, MNRAS, 468, 509

Grand R. J. J., Kawata D., Cropper M., 2012, MNRAS, 426, 167

Grasha K. et al., 2015, ApJ, 815, 93

Grasha K. et al., 2017a, ApJ, 840, 113

Grasha K. et al., 2017b, ApJ, 842, 25

Julian W. H., Toomre A., 1966, ApJ, 146, 810

Kaleida C., Scowen P. A., 2010, AJ, 140, 379

Korchagin V., Kikuchi N., Miyama S. M., Orlova N., Peterson B. A., 2000, ApJ, 541, 565

Kormendy J., Norman C. A., 1979, ApJ, 233, 539

Kroupa P., 2001, MNRAS, 322, 231

Krumholz M. R. et al., 2015, ApJ, 812, 147

Landy S. D., Szalay A. S., 1993, ApJ, 412, 64

Lee J. C. et al., 2009, ApJ, 706, 599

Lin C. C., Shu F. H., 1964, ApJ, 140, 646
Lindblad B., 1963, StoAn, 22

Louie M., Koda J., Egusa F., 2013, ApJ, 763, 94

Martínez-García E. E., González-Lópezlira R. A., Bruzual-A G., 2009, ApJ, 694,512

Messa et al., 2018, MNRAS, 473, 996

Olivares E. F. et al., 2010, ApJ, 715, 833

Peebles P. J. E., 1980, Proceedings of the 9th Texas Symposium on Relativistic Astrophysics, Munich, West Germany, December 14-19, 1978, New York Academy of Sciences. New York, p. 161

Pettitt A. R., Tasker E. J., Wadsley J. W., 2016, MNRAS, 458, 3990

Rand R. J., 1995, AJ, 109, 2444

Roberts W. W., 1969, ApJ, 158

Sabbi E. et al., 2018, ApJS, 235, 23

Sakhibov F. K., Smirnov M. A., 2004, Astron. Rep, 48, 995

Sánchez-Gil M. C., Jones D. H., Pérez E., Bland-Hawthorn J., Alfaro E. J., O'Byrne J., 2011, MNRAS, 415, 753

Scheepmaker R. A., Lamers H. J. G. L. M., Anders P., Larsen S. S., 2009, A\&A, 494, 81

Schinnerer E. et al., 2017, ApJ, 836, 62

Schlafly E. F., Finkbeiner D. P., 2011, ApJ, 737, 103

Sellwood J. A., 2011, MNRAS, 410, 1637

Sellwood J. A., Carlberg R. G., 1984, ApJ, 282, 61

Sheth K. et al., 2010, PASP, 122, 1397

Shu F. H., 2016, ARA\&A, 54, 667

Smajić S., Moser L., Eckart A., Busch G., Combes F., García-Burillo S., Valencia-S. M., Horrobin M., 2015, A\&A, 583, A104

Sofue Y., 1996, ApJ, 458, 120

Sofue Y., 1997, PASJ, 49, 17

Tamburro D., Rix H.-W., Walter F., Brinks E., de Blok W. J. G., Kennicutt R. C., Mac Low M.-M., 2008, AJ, 136, 2872

Tonry J. L., Dressler A., Blakeslee J. P., Ajhar E. A., Fletcher A. B., Luppino G. A., Metzger M. R., Moore C. B., 2001, ApJ, 546, 681

Toomre A., Toomre J., 1972, ApJ, 178, 623

Vogel S. N., Kulkarni S. R., Scoville N. Z., 1988, Nature, 334, 402

Zackrisson E., Rydberg C.-E., Schaerer D., Östlin G., Tuli M., 2011, ApJ, 740, 13

Zimmer P., Rand R. J., McGraw J. T., 2004, ApJ, 607, 285

This paper has been typeset from a $\mathrm{T}_{\mathrm{E}} \mathrm{X} / \mathrm{L} \mathrm{T}_{\mathrm{E}} \mathrm{X}$ file prepared by the author. 